Revista del Centro de Investigación de la Universidad La Salle

Vol. 13, No. 49, enero-junio 2018: 91-103

DOI : https://doi.org/10.26457/recein.v13i49.1740

\title{
Monitoreo de la materia orgánica disuelta de un reactor biológico secuencial de lodo activado a través de espectroscopía de fluorescencia
}

\author{
Miroslava de los Ángeles Mier Quiroga ${ }^{1}$ \\ Universidad Autónoma del Estado de México (México) \\ Mario Esparza Soto \\ Universidad Autónoma del Estado de México (México) \\ Mercedes Lucero Chávez \\ Universidad Autónoma del Estado de México (México)
}

Fecha de recepción: 26 de septiembre de 2018

Fecha de aceptación: 08 de noviembre de 2018

Disponible en línea: 30 de noviembre de 2018

\section{Resumen}

Los efluentes de reactores de tratamiento biológico contienen una mezcla heterogénea de materia orgánica disuelta (MOD), la cual es de gran importancia conocer debido al impacto que tiene sobre el proceso (calidad del efluente). En esta investigación los componentes de la MOD en un reactor biológico secuencial de lodos activados (RBS-LA) fue caracterizada usando matrices de fluorescencia excitación/emisión (EEMs, por sus siglas en inglés) para

\footnotetext{
${ }^{1}$ E-mail: mirosmier@gmail.com
}

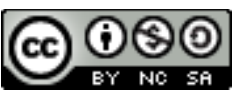

Revista del Centro de Investigación. Universidad La Salle por Dirección de Posgrado e Investigación. Universidad La Salle Ciudad de México se distribuye bajo una Licencia Creative Commons Atribución- 
Mier Quiroga, M.; Esparza Soto, M.; Lucero Chávez, M.

identificar actividad microbiana. El principal objetivo de esta investigación fue monitorear la transformación de la MOD dentro de un reactor de 100 L SBR-LA, el cual fue operado a un tiempo de retención hidráulico de 8 horas, siendo alimentado por agua residual cruda (diluida para elevar el $\mathrm{pH}$ ) proveniente de la industria chocolatera. LA MOD fue monitoreada utilizando espectroscopía de fluorescencia a través de cual se obtuvieron los picos máximos en las EEMs. El análisis visual que se llevó a cabo de las EEMs mostró en el efluente pares de picos que de acuerdo con la literatura se pueden atribuir a sustancias proteicas y sustancias húmicas. Dichas sustancias no se observaron en las EEMs del influente por lo cual se atribuyen a los productos microbianos solubles generados dentro del reactor. Se puede concluir que el análisis visual llevado a cabo es cualitativo y no cuantitativo ya que la intensidad de fluorescencia es mayor en el efluente mientras que la demanda química de oxígeno es menor.

Palabras clave: Productos microbianos solubles, matriz excitación/emisión, tratamiento aerobio. 


\section{Abstract}

The effluents of biological treatment reactors contain a heterogeneous mixture of dissolved organic matter (DOM), which is of great importance to know due to the impact it has on the process (effluent quality). In this research, the components of DOM in a Sequential Batch Reactor of activated sludge (SBR-AS) were characterized using excitation/emission fluorescence matrix (EEM) to describe microbial activity. The main objective was to monitor the transformation of the DOM into a reactor of $100 \mathrm{~L} \mathrm{SBR-AS,} \mathrm{which} \mathrm{was} \mathrm{operated} \mathrm{at} \mathrm{a}$ hydraulic retention time of 8 hours, being fed by residual raw water (diluted to raise the $\mathrm{pH}$ ) from the chocolate industry. The DOM was monitored using fluorescence spectroscopy through which maximum peaks were obtained in the EEMs. Visual analysis of EEMs showed in the effluent pairs of peaks that according to the literature can be attributed to protein-like and humic-like substances. These substances were not observed in the influent's EEMs, so they are attributed to the soluble microbial products generated within the reactor. It can be concluded that the visual analysis carried out is qualitative and not quantitative since the fluorescence intensity is greater in the effluent while the chemical oxygen demand is lower.

Keywords: soluble microbial products, excitation/emission matrix, aerobic treatment 


\section{Introducción}

La característica principal de los sistemas de tratamiento de agua residual biológicos (aerobios o anaerobios) es remover la materia orgánica que llega a sus influentes (Metcalf \& Eddy, 2003), por otro lado, los efluentes de los sistemas de tratamiento biológico contienen una mezcla de compuestos orgánicos solubles tales como polisacáridos, proteínas, ácidos orgánicos, etc. producto de los residuos de los microorganismos. Debido a esto, la materia orgánica disuelta (MOD) causa una gran preocupación en términos del proceso de operación y el control de la calidad del efluente y es necesario conocer las características que presenta al entrar y al salir del sistema.

Alta concentración de MOD ya sea como productos microbianos solubles (PMS) o sustancias poliméricas extracelulares pueden causar un rendimiento deficiente en sistemas de tratamiento biológicos convencionales (Chen et al., 2003). Las características de la MOD están afectadas por parámetros operacionales tales como: tiempo de retención celular (TRC), tiempo de retención hidráulico (TRH), carga orgánica, y tasa comida/microorganismos (Tahir et al., 2017).

La luz puede ser absorbida y emitida por ciertos compuestos y a este proceso se lo denomina luminiscencia. Existen dos tipos: fluorescencia y fosforescencia. La fluorescencia es un fenómeno físico mediante el cual ciertas sustancias absorben energía en forma de radiación electromagnética emitiéndola en una longitud de onda mayor en un periodo de tiempo muy corto (Albani, 2007). La fluorescencia es el resultado de una serie de procesos que ocurren en ciertas moléculas llamadas fluoroforos. Dichas moléculas son generalmente orgánicas poliaromáticas o heterociclos.

Hasta ahora se han empleado varias herramientas espectroscópicas para desenredar la compleja estructura de la MOD en los sistemas de tratamiento biológico ya que la materia orgánica absorbe luz en un amplio rango de longitudes de onda. Como resultado, la absorbancia de luz es un indicador semi cuantitativo de la concentración de la materia orgánica en el agua (Korshin et al., 1997). 
Monitoreo de la materia orgánica disuelta de un reactor biológico secuencial de lodo activado a través de espectroscopía de fluorescencia

Dentro de las diferentes herramientas espectroscópicos, la fluorescencia ha sido aplicada recientemente a los sistemas de tratamiento para caracterizar cambios en la MOD. Debido a que es fácil de obtener y cuenta con la habilidad de proveer las características químicas de la mayor parte de la MOD (Korak et al., 2014; Tahir et al., 2017; Cohen et al., 2014; Bridgman et al., 2013).

El principal objetivo de esta investigación fue monitorear la transformación de la MOD dentro de un reactor piloto de 100 L RBS-LA el cual fue alimentado por agua cruda residual proveniente de la industria chocolatera. La MOD fue monitoreada utilizando espectroscopía de fluorescencia a través de cual se realizó el análisis visual de las EEMs del influente y del efluente para determinar los picos propuestos por Chen, et al (2003).

\section{Metodología}

A. Operación y monitoreo del reactor piloto RBS-LA

Se operó un RBS-LA con un volumen de $100 \mathrm{~L}$ previamente estabilizado para otra investigación con un TRC de $11 \pm 4.39$ días (tiempo establecido a partir de los cálculos realizados por Mier-Quiroga (2016)). El RBS-LA trató agua proveniente de la industria chocolatera con una concentración de demanda química de oxígeno soluble (DQOs) en su influente variable. El agua residual de la industria chocolatera que se utilizó en esta investigación es entregada con un $\mathrm{pH}$ ácido $(5.1 \pm 1.02)$. Debido a que los sistemas de tratamiento biológicos deben operar con un $\mathrm{pH}$ cercano a 7 fue necesario diluir el agua residual con el efluente del RBS-LA, de esta forma se evitó la adición de compuestos como $\mathrm{NaOH}$ para elevar el $\mathrm{pH}$. El porcentaje de dilución se modificó con cada lote de agua residual (1000 L) debido al cambio de $\mathrm{pH}$ del agua residual cruda.

El TRH fue de 8 horas. El ciclo del RBS-LA consistió en una fase de aireación y mezcla de 6 horas, una fase de sedimentación de 1 hora, una fase de purga del efluente 0.5 horas y una fase de llenado 0.5 horas. Se realizaron tres ciclos por día. El RBS-LA fue operado mediante un controlador diseñado para modificar los TRH por Mier-Quiroga (2016). 
El RBS-LA había sido operado con TRH de 8 horas desde 12 meses previos a esta investigación y se ha monitoreado periódicamente ( $\mathrm{pH}$ y DQOs).

El aireado y mezcla del RBS-LA para mantener $2 \mathrm{mg} / \mathrm{L}$ de oxígeno disuelto (OD) se llevó a cabo con un aireador marca GAST modelo Regenair R3 105-1. El aire fue suministrado al reactor mediante un difusor previamente diseñado y construido.

Las muestras para esta investigación se tomaron durante la fase de sedimentación 10 minutos previos al final de un ciclo (cada 24 horas) y en el influente del RBS-LA. Se tomaron 29 muestras del efluente del RBS-LA; al haber tomado 13 muestras del efluente se decidió muestrear el influente por lo que sólo se analizaron16 muestras de éste. Las muestras fueron tomadas durante un periodo de 4 meses en días aleatorios.

Todas las muestras fueron filtradas con filtros de fibra de vidrio (GF/C, Whatman). Las muestras filtradas fueron colectadas y almacenadas en viales ámbar, acidificadas a un $\mathrm{pH}$ de $2 \mathrm{y}$ almacenadas a $4^{\circ} \mathrm{C}$ hasta ser analizadas.

De todas las muestras se obtuvo la DQOs y se realizó su EEM. Se generó la EEM de corrección del blanco, realizándolo con agua ultrapura obtenida del desionizador Milliepore marca modelo Millie-Q Plus®.

\section{B. Métodos analíticos}

La DQOs fue determinada con un kit comercial colorimétrico de reflujo cerrado (rango alto 0-1500 mg/L DQO, Hach, Co.). Para estandarizar las muestras en este estudio y poder comparar las EEMs se diluyeron las muestras a $5 \mathrm{mg} / \mathrm{L}$ DQOs (se eligió $5 \mathrm{mg} / \mathrm{L}$ debido a que fue la dilución en donde las muestras tanto del influente como del efluente entraron en el rango de fluorescencia).

Todas las matrices EEM fueron obtenidas en un espectrómetro de fluorescencia (Modelo LS-55, Perkin Elmer, USA) equipado con una lámpara de Xenon de 150 watts como fuente de excitación. El espectrómetro se controlado con el software WINLAB en una computadora de escritorio. Las EEMs consisten en 45 escaneos de emisión (250-600 nm) recolectados en longitudes de onda de excitación de 200 a 420 nm en incrementos de 5 nm. 
Monitoreo de la materia orgánica disuelta de un reactor biológico secuencial de lodo activado a través de espectroscopía de fluorescencia

La abertura de excitación/emisión se estableció en $10 \mathrm{~nm}$. La velocidad de escaneo se fijó en $1200 \mathrm{~nm} / \mathrm{min}$.

Todos los archivos generados por WINLAB como EEMs fueron exportados a Excel ${ }^{\circledR}$. Todos los libros de Excel generados fueron diseñados para restar el blanco a las EEMs y así eliminar las dispersiones de Rayleigh y Raman. Se realizó un análisis visual por medio del cual se detectó la mayor intensidad de fluorescencia (pico máximo) y su localización en las EEM.

\section{Resultados y Discusión}

\section{A. Desempeño del RBS-LA}

Debido a que existieron variaciones en la calidad del agua de la industria chocolatera la DQOs del influente se mantuvo en $523.94 \pm 166.25 \mathrm{mg} / \mathrm{L}$. A pesar de un influente variable el desempeño del RBS-LA se mantuvo estable ya que en su efluente la DQOs se obtuvo en $82.86 \pm 52 \mathrm{mg} / \mathrm{L}$ con un porcentaje de remoción del 75\%. El pH del influente debido a la dilución realizada para elevarlo se mantuvo en el influente en $7.06 \pm 0.37 \mathrm{y}$ en el efluente en $8.64 \pm 17$

Los sólidos suspendidos (SS) dentro del RBS-LA no fueron analizados durante esta investigación porque ya se tiene información recolectada previa durante 250 días en donde los SS dentro del RBS-LA se mantuvieron variables debido al cambio en el influente, pero los SS en el efluente se mantuvieron en $55.23 \pm 15.46 \mathrm{mg} \mathrm{SS} / \mathrm{L}$, independientemente de la concentración de SS del licor mixto.

B. Análisis visual de las matrices de excitación/emisión

Para llevar a cabo un correcto análisis visual de espectroscopía de fluorescencia se tomaron en cuenta las propiedades más importantes de la fluorescencia que son la localización de los picos de exctiación y emisión (sus respectivas longitudes de onda) y la intensidad máxima de fluorescencia. Si conocemos estos aspectos de la muestra analizada se pueden identificar cualitativamente los fluoróforos presentes en la muestra. 
Las figuras 1 y 2 muestran las EEMs representativas del influente y efluente del RBSLA respectivamente, mientras que las tablas 1 y 2 muestra los promedios de las intensidades de fluorescencia (IF) expresadas en unidades arbitrarias de fluorescencia (UA) y las longitudes de onda de excitación y emisión de los picos más representativos (picos $\mathrm{A}, \mathrm{B}, \mathrm{C}$, D y E).

Debido a que el influente fue diluido con el efluente del RBS-LA se debe considerar que gran parte de su fluorescencia se puede atribuir a los PMS que se generaron dentro del reactor y no a fluorescencia presente en el agua residual cruda. La EEM del influente muesta poca IF (250 UA) respecto a la del efluente (650 UA) por lo que el primer análisis visual indica que el aumento de la fluorescencia y el cambio de forma de la EEM es debido a producción microbiana de reciente creación.

La localización de los picos A, B, C y D se compararon con las zonas establecidas en la literatura (Chen et al., 2003). Los picos A y B se identificaron como sustancias proteicas atribuidas a los PMS (triptófano) (Baghot et al., 2011). Los pico A y B tiene una mayor IF en el efluente que en el influente, esto se puede atribuir a que se ha establecido su presencia cuando existe una alta actividad microbiana (Tahir et al., 2017). El pico D por otro lado, al ubicarse dentro de la zona de las sustancias húmicas, se ha establecido como representativo de MOD refractaria resultado de la biodegradación de materia vegetal y/o animal.

Tabla 1

Promedios y desviación estándar de los picos A, B y C del influente del RBS-LA.

\begin{tabular}{|c|c|c|c|c|c|c|c|c|c|c|c|c|}
\hline & \multicolumn{3}{|c|}{ A } & \multicolumn{3}{|c|}{ B } & \multicolumn{3}{|c|}{ C } & \multicolumn{3}{|c|}{ D } \\
\hline & ex & em & IF & ex & em & IF & ex & em & IF & ex & em & IF \\
\hline PROM. & 219.06 & 364.7 & 194.58 & 277.5 & 364.82 & 65.4 & - & - & - & 328.75 & 424.81 & 50.901 \\
\hline n. & 16 & 16 & 16 & 16 & 16 & 16 & 16 & 16 & 16 & 16 & 16 & 16 \\
\hline
\end{tabular}


Monitoreo de la materia orgánica disuelta de un reactor biológico secuencial de lodo activado a través de espectroscopía de fluorescencia

Tabla 2

Promedios y desviación estándar de los picos $A, B, C$, D y E del efluente del RBS-LA

\begin{tabular}{|c|c|c|c|c|c|c|c|c|c|c|c|c|c|c|c|}
\hline & \multicolumn{3}{|c|}{ A } & \multicolumn{3}{|c|}{ B } & \multicolumn{3}{|c|}{ C } & \multicolumn{3}{|c|}{ D } & \multicolumn{3}{|c|}{$E$} \\
\hline & ex & em & IF & ex & em & IF & ex & em & IF & ex & em & IF & ex & em & IF \\
\hline PROM & 223.2 & 367.1 & 467.4 & 295.0 & 378.2 & 204.7 & 233 & 418. & 386.7 & 331 & 414.3 & 244.1 & 277.5 & 417.9 & 211.0 \\
\hline n. & 29 & 29 & 29 & 29 & 29 & 29 & 29 & 29 & 29 & 29 & 29 & 29 & 29 & 29 & 29 \\
\hline
\end{tabular}

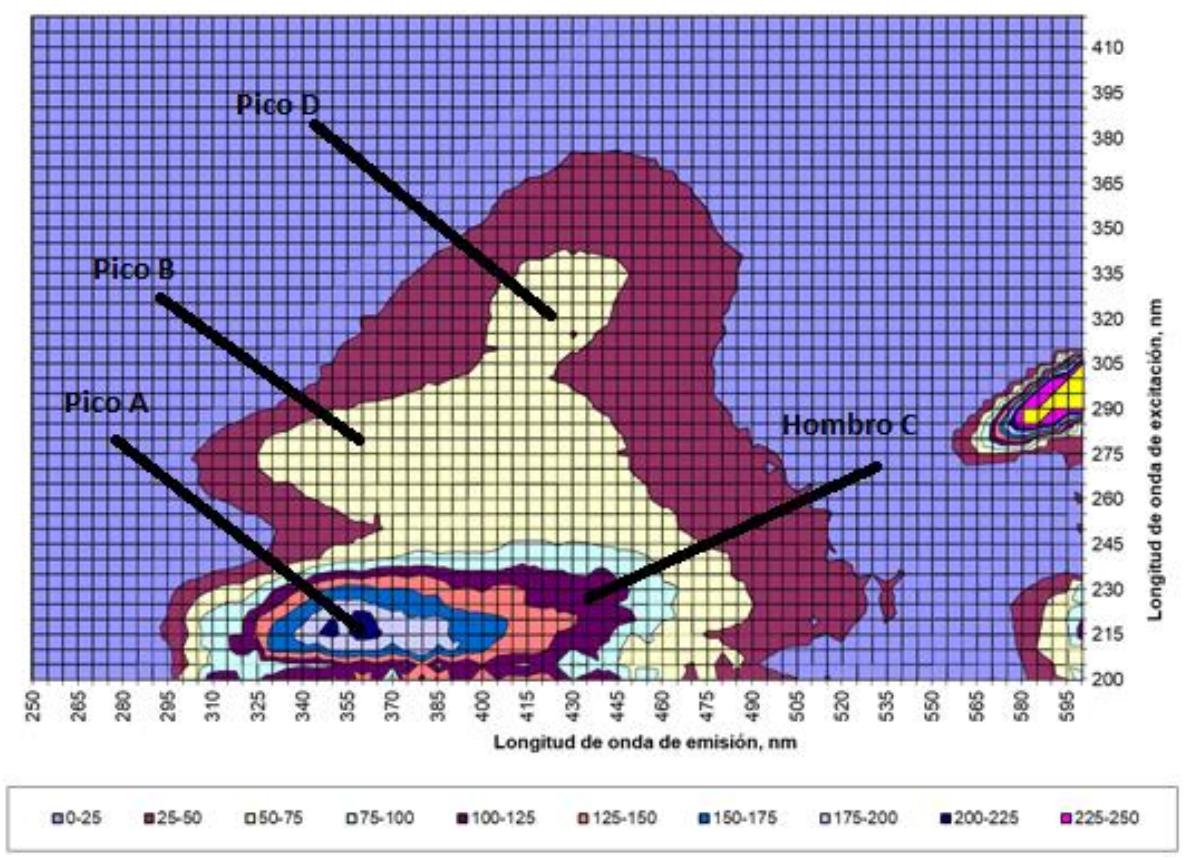

Figura 1. Matriz excitación/emisión representativa del influente del RBS-LA. Cada línea representa 25 U.A. 


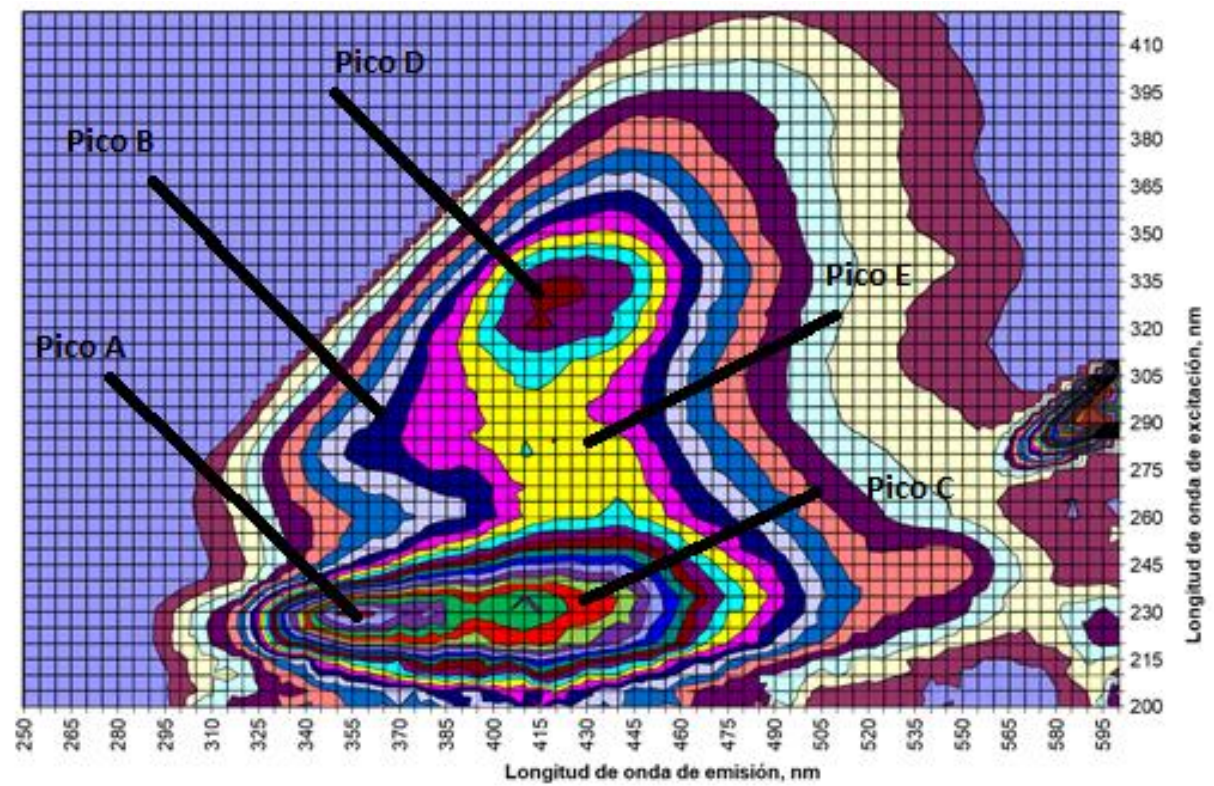

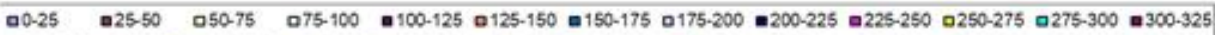

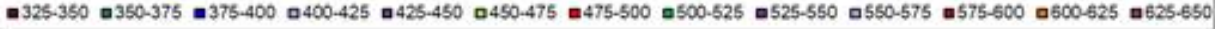

Figura 2. Matriz excitación/emisión representativa del efluente del RBS-LA. Cada línea representa 25 U.A.

La humificación microbiana en los lodos activados y/o en el proceso de digestión se ha encontrado en diferentes estudios. Este proceso está típicamente acompañado por la producción de más estructuras aromáticas complejas, las cuales son más resistentes a la degradación microbiana (Tahir et al., 2017; McKnight et al., 2001; Stedmon et al., 2003).

El hombro C presente en el influente se ha reportado como un pico caracterizado por acompañar al pico D y establece presencia de sustancias húmicas (Komatuso et al., 2018). En el efluente se muestra el pico $\mathrm{C}$ ya no como hombro, esto se atribuye a que el efluente presenta mayor IF en la zona atribuida a sustancias húmicas. Se necesita un metodo analítico diferente para poder aislar al pico $\mathrm{C}$ y establecer sus propiedades y origenes.

El pico E solo visible en el efluente del RBS-LA y se encuentra en la zona establecida por Chen et al. (2003) como la zona de sustáncias húmicas y fúlvicas. A pesar de que las intensidades de fluorescencia del influente se ven afectadas por las del efluente debido a la mezcla producida con el agua cruda de la industria chocolatera, se tiene que resaltar que el pico E no se encuentra presente en las EEMs del influente, por lo tanto, es necesario abordar otro tipo de técnica de análisis para conocer el origen de este pico. 


\section{Conclusiones}

La MOD del RBS-LA fue caracterizada mediante espectroscopía de fluorescencia en su influente y efluente. Después del tratamiento las intensidades de fluorescencia de todos los picos se incrementó y apareció un pico no observado en el influente lo que indica que los cambios en las EEMs son producidos por actividad microbiana resultando en tener un aumento en los PMS.

Con el análisis visual y establecimiento de los picos máximos se encontró que existe presencia de sustancias proteicas (triptófano), sustancias húmicas con su poible hombro (pico C) que se atribuyen a la producción microbiana.

El análisis visual de la espectroscopía de fluorescencia se puede establecer con los resultados obtenidos como un análisis cualitativo, ya que la DQOs del efluente es menor que la DQOs del influente, mientras que las intensidades de fluorescencia se comportan de manera inversa. Si fuera requerido un análisis en donde se pueda comparar la MOD cuantitativamente con ayuda de la fluorescencia, es necesario hacer otro tipo de análisis como la literatura los ha establecido para aguas naturales.

Debido a lo previamente mencionado sobre la influencia de los parámetros operacionales se recomienda modificarlos y al compararlos se puedan conocer y monitorear de manera más clara la producción de las sustancias húmicas y proteicas. 


\section{Referencias}

Albani, Jihad, R. (2007). Principles and Applications of Fluorescence Spectroscopy. Blackwell Science.

Baghoth, S., Sharma, S., \& Amy, G. (2011). Tracking natural organic matter (NOM) in a drinking water treatment plant using fluorescence excitation-emission matrices and PARAFAC. Water Research, 797-809.

Bridgeman, J., Baker, A., Carliell-Marquet, C., Carstea, E., (2013). Determination of changes in wastewater quality through a treatment works using fluorescence spectroscopy. . Environment Science and Technology 34, 3069-3077.

Chen, W., Westerhoff, P., Leenheer, J. A., \& Booksh, K. (2003). Fluorescence Excitation-Emission Matrix Regional Integration to Quantify Spectra for Dissolved Organic Matter. Environment Science and Technology 37, 5701-5710.

Cohen, E., Levy, G.J., Borisover, M., (2014). Fluorescent components of organic matter in wastewater: Efficacy and selectivity of the water treatment. Water Research. $55,323-334$.

Komatuso, K., Imai, A., Kawasaki, N., (2018). Comparison between humic-like peaks in excitation-emission matrix spectra and resin-fractionated humica substances in aquatic environments. Limnology, 1-12.

Korak, J. A., Dotson, A. D., \& Summers, R. S. (2014). Critical analysis of commonly used fluorescence metrics to characterize dossolved organic matter. Water Research 49, 327-338.

Korshin, G. V., Li, C.-W., \& Benjamin, M. M. (1997). Monitoring the properties of natural organic matter through UV spectroscopy: a consistent theory. Water Research 31, 1787-1795.

McKnight, D., Boyer, E., Westerhoff, P., Doran, P., Kulbe, T., Andersen, D., (2001). Spectrofluorometric characterization of dissolved organic matter for indication of 
Monitoreo de la materia orgánica disuelta de un reactor biológico secuencial de lodo activado a través de espectroscopía de fluorescencia

precursor organic material and aromaticity. Limnology and Oceanography, 46, $38-48$.

Metcalf \& Eddy (2003). Wastewater engineering: Treatment and reuse. united states of america: McGraw-Hill.

Mier-Quiroga, M. (2016). Mejoramiento de la sedimentación de un lodo activado mediante la adición de magnesio. Toluca, CIRA: Universidad Autónoma del Estado de México.

Stedmon, C., Markager, S., Bro, R. Tracing dissolved organic matter in aquatic environments using a new approach to fluorescence spectroscopy. Marine Chemestry 82, 239-254.

Tahir, M., Jinwoo, C., \& Jin, H. (2017). Spectroscopic descriptors for dynamic changes of soluble microbial products from activated sludge at different biomass growth phases under prolonged starvation. Water Research 123, 751-760. 